\title{
Analysis of Symmetry of Weight-Bearing in Athletes With and Without Low Back Pain during Gait
}

\author{
${ }^{1,2}$ Meissam Sadeghisani ${ }^{*},{ }^{2}$ Vahid Sobhani, ${ }^{3}$ Hadi Azimi, ${ }^{4}$ Mohammad Taghi Karimi \\ ${ }^{1}$ Shahid Beheshti University of Medical Sciences, Tehran, Iran. ${ }^{2}$ Exercise Physiology Research Center, Baqiyatallah \\ University of Medical Sciences, Tehran, IR Iran. ${ }^{3}$ Department of English Language Teaching, School of Medicine, Shahid \\ Beheshti University of Medical Sciences, Tehran, Iran. ${ }^{4}$ Isfahan University of Medical Sciences, Isfahan, Iran.
}

\begin{abstract}
Background. Low Back Pain (LBP) is a prevalent phenomenon in athletes and asymmetrical loading on the limbs has been proposed as a risk factor related to this disease. Objectives. The purpose of the present study was to compare the asymmetry of loading applied on the legs between men with and without LBP during gait. Methods. A total of 40 participants, comprising 20 men with non-specific LBP who practiced martial arts and 20 men without LBP, participated in the study. The participants walked in gait lab and forces applied on the legs were recorded in three dimensions using a force plate. Also, asymmetry of the forces applied on the dominant and non-dominant limbs was measured. Results. The Asymmetry Indexes (ASI) of the first, second, and third peaks of vertical forces were respectively $3.1 \%, 3.4 \%$, and $4.1 \%$ for normal participants and $4.2 \%, 4.2$, and 2.1 for the participants who had LBP ( $>0.05$ ). Conclusion. It can be concluded that martial arts athletes with LBP apply symmetrical loads on the lower extremities, similar to healthy people.
\end{abstract}

KEY WORDS: Low Back Pain, Asymmetry, Gait, Weight-Bearing.

\section{INTRODUCTION}

Among musculoskeletal dysfunctions, low Back Pain (LBP) is known as the most common health problem worldwide $(1,2)$. Depending on the population, annual prevalence is reported $15-$ $45 \%$ (3). It accounts for 5 to $8 \%$ of athletic injuries, making it one of the most challenging to diagnose and treat injuries for the clinicians (4).

LBP in Iran, as with other countries, imposes heavy economic burden on the community, government, and family (5). Multifactorial risk factors, including bio-psychosocial, are associated with this syndrome (6). Among these risk factors, biomechanical factors have been proposed as the most important cause of the disease (7). By identifying and correcting the impaired biomechanical situation, which are associated with LBP, clinicians would be able to help the LBP patients, especially those who do sport activities, to achieve healthy condition (6).

With regard to this, various mechanical characteristics have been suggested as causes of LBP, including impaired muscle endurance (8), uncontrolled lumbopelvic motion (9-11), lower limbs range of motion deficit (12-14), and prolonged sitting and standing (15). Some mechanical risk factors of LBP have also been investigated in Iran (16-19). Some of these

*. Corresponding Author:

Meissam Sadeghisani

E-mail: sadeghi.m@sbmu.ac.ir 
contributing factors are also investigated in athletes who had $\operatorname{LBP}(4,20)$.

The asymmetrical loading applied on the legs during functional activities is a biomechanical risk factor, which could contribute to developing symptoms in patients with musculoskeletal disorders (21-24). Some authors believe that asymmetrical loading on the limbs during functional and recreational activities, such as a sport activity, may be associated with asymmetrical loading applied on the lumbar spine tissues $(21,22,25,26)$. If applied on the tissues during habitual or functional activities, asymmetrical loading could lead to micro-trauma and eventually LBP. Therefore, study of the pattern of loads applied on the legs in athletes with musculoskeletal symptoms, especially LBP, seems to be critical.

Martial arts are sports activities which are popular among many around the world (27), especially among Iranian people. Although no exact statistics on the number of Iranian athletes who do these sports exist, a significant number of people participate in such activities in other countries (27). These sports are also among the fields of Olympic Games which normally win medals for Iran. Based on the clinical examination, LBP was found to be a prevalent musculoskeletal disorder among Iranian athletes who practice Martial arts.

Our literature review revealed that, like those suffering from LBP, some risk factors hypothesized to be related to the symptoms (28) were examined in people with LBP who practiced martial arts, as well. Based on our literature review, the pattern of loading in LBP patients was examined in three studies during standing and gait $(22,25,26)$. In Zahraee et al. study, only women with LBP were enrolled (25). However, no reported study has yet investigated the pattern of forces applied on the legs in martial arts athletes who have LBP. The the present study was conducted to compare the asymmetry of loading applied on the legs between martial arts athletes with LBP and people without LBP during gait.

\section{MATERIALS AND METHODS}

Participants. Two groups, one with persistent LBP and one without a history of LBP, participated in the current study: 20 men with non-specific, chronic LBP and 20 men with no previous LBP. All athletes with persistent LBP had history of lumbar spine pain symptom for more than 3 months with non-specific LBP diagnosis (29) made by physicians. They were athletes who practiced martial arts at least two sessions in a week. The healthy participants were male students of Isfahan University. The control participants were examined by a physician for any symptoms or dysfunctions in their lumbar, pelvis, thoracic and lower extremities to rule out those who had lumbar spine impairments. The exclusion criteria for both groups were marked kyphosis or scoliosis, spinal tumor or other serious diseases, rheumatology or immunological diseases, discrepancy of legs' length (30), previous surgery, and/or degenerative joint disease in a lower limb or spine. Eligible individuals were enrolled after signing an informed consent and the study procedure was approved by Ethics Committee of Baqiyatallah University of Medical Sciences.

Test. Participants were asked to walk in the gait lab at the Faculty of Rehabilitation, Isfahan University of Medical Sciences. Participants walked a $20-\mathrm{m}$ distance with comfortable speed from which six successful trails were selected for each leg (three trials for the dominant limb and three trials for the non-dominant limb) (26).

Instrumentation and data processing. To record the forces applied on the legs, the Kistler force plate was used in the Faculty of Rehabilitation, Isfahan University of Medical Sciences. The data were recorded at $120-\mathrm{Hz}$ frequency. After data collection, all the data were filtered using a Butterworth low pass filter with a cut-off frequency of $10 \mathrm{~Hz}$. Then, peaks of the forces in vertical, anterior-posterior, and medial-lateral directions were determined for each test of legs. The peaks of the forces were extracted using QTM software. The asymmetrical force applied on the legs in each direction was determined based on the asymmetry index (ASI) proposed by Herzog et al. (31) and others (24-26, 31). For the ASI calculation, we have:

$$
\mathrm{ASI}=\frac{\mathrm{X}-\mathrm{Y}}{1 / 2(\mathrm{X}+\mathrm{Y})} \cdot 100
$$

Where $\mathrm{X}$ represents the force applied on the dominant side and $\mathrm{Y}$ is the same force applied on the non-dominant side. Low value of ASI reveals that the asymmetry between sides is small and vice versa. Figure 1 indicates a sample of forces applied on the leg in one of the participants. 
Statistical analysis. For all steps of data analysis, SPSS, version 21, was used. Initially, normality of data was evaluated running Kolmogorov-Smirnov test. Since the data were distributed normally, we used independent t-test for comparison between groups.

\section{RESULTS}

As Table 1 shows, individuals with and without LBP were not different with regard to all demographic characteristics ( $\mathrm{p}>0.05)$.

The results presented in Table 2 indicate that there was no significant difference between the two groups in stride length, velocity, and cadence of gait for the dominant leg ( $>>0.05)$.

Table 3 also summarizes the mean values of the ASI for the forces applied on the legs in three directions. For the vertical direction, no significant differences in the ASI of the forces were observed between the groups during heel strike (Fz1), mid stance, and push off (Fz3) phases of the test ( $p>0.05)$. For anterior-posterior direction, the ASI of the anterior force (Fx1) applied on the ground was $12.8 \pm 8.2$ in the healthy individuals and $14.4 \pm 8.7$ in the patients $(p=0.577)$. Also, the mean value of the ASI in the posterior direction (Fx2) in the patients was $7.3 \pm$ 8.8 vs. $6.2 \pm 6.5$ in the healthy individuals $(\mathrm{p}=0.670)$.

The ASI of the peak of medial-lateral force (Fy) was calculated to be $11.2 \pm 10 \%$ and $15.9 \pm$ $21.8 \%$ in patients with non-specific LBP and healthy participants, respectively $(\mathrm{p}=0.402)$.

Table 1. Demographic characteristics of People without and with LBP, $n=20$, and Mean $( \pm S D)$

\begin{tabular}{lcccc}
\hline \multicolumn{1}{c}{ Variable } & without LBP & with LBP & DF & p \\
\hline Age (years) & $30( \pm 6.3)$ & $29.1( \pm 5.9)$ & 38 & 0.628 \\
Height $(\mathrm{m})$ & $1.76( \pm 0.07)$ & $1.76( \pm 0.06)$ & 38 & 0.791 \\
Weight $(\mathrm{kg})$ & $76.8( \pm 11.3)$ & $74.6( \pm 11.8)$ & 38 & 0.561 \\
MBI $\left(\mathrm{kg} / \mathrm{m}^{2}\right)$ & $24.7( \pm 3.2)$ & $23.9( \pm 3.6)$ & 38 & 0.443 \\
Duration of LBP (month) & NA & $20( \pm 18.2)$ & & \\
Pain intensity & NA & $2.7( \pm 2.4)$ & & \\
Oswestry score & NA & $21.1( \pm 7.8)$ & & \\
Habitual physical activity score & NA & $5.8( \pm 1.3)$ & & \\
\hline
\end{tabular}

Table 2. Spatiotemporal variables in the two groups of study (dominant leg)

\begin{tabular}{lccc}
\hline & Patients group & Healthy group & P value \\
\hline Stride length $(\mathrm{m})$ & $1.2 \pm 0.8$ & $1.28 \pm 0.12$ & 0.816 \\
Velocity $(\mathrm{m} / \mathrm{s})$ & $1.1 \pm 0.12$ & $1.1 \pm 0.2$ & 0.427 \\
Cadence $($ steps $/ \mathrm{min})$ & $104.5 \pm 6.7$ & $103.1 \pm 13.2$ & 0.732 \\
\hline
\end{tabular}

Table 3. The means of asymmetrical values of spatiotemporal and force parameters of healthy and patient groups

\begin{tabular}{c|c|c|c|c|c|c}
\hline variable & Fz1 & Fz2 & Fz3 & Fy & Fx1 & Fx2 \\
\hline Patients group & $4.2 \pm 2.82 .8$ & $4.23 \pm 3.9$ & $2.1 \pm 1.8$ & $11.6 \pm 9.8$ & $14.4 \pm 8.7$ & $7.3 \pm 6.8$ \\
Healthy group & $3.3 \pm 2.7$ & $3.8 \pm 3$ & $2.2 \pm 1.8$ & $7.6 \pm 5.8$ & $12.3 \pm 9.4$ & $10.6 \pm 10.0$ \\
p & 0.445 & 0.749 & 0.956 & 0.131 & 0.474 & 0.279 \\
\hline
\end{tabular}

\section{DISCUSSION}

The LBP has a bio-psychosocial nature (6), with associated environmental and individual factors (2). Mechanical risk factors have been proposed as the most contributing factor $(7,15,32$, 33) and are therefore addressed in many studies.

Asymmetrical load bearing is a mechanical risk factor which has already been investigated in people with musculoskeletal disorders (22-25). A significant asymmetrical loading on the limbs could be associated with an asymmetrical stress in bilateral tissues, which could lead to tissue injuries and therefore musculoskeletal pain syndrome. In LBP, significant difference in load applied on the lower limbs is likely to result in symmetrical stress in the lumbar spine tissues. If this situation is 
frequently repeated, the LBP will happen. Although kinematic variables reveal asymmetrical characteristics of motions in LBP patients as compared to healthy people $(28,34-36)$, little information has been obtained about the symmetry of kinetic characteristics of motion in LBP patients.

Gait is an important functional activity repeated frequently throughout daily activities. Asymmetrical loading on the limbs during gait may result in different musculoskeletal pain syndromes such as $\operatorname{LBP}(21,24,25)$. To date, symmetry of loading in people with LBP, to the best of our knowledge, has been investigated only in two studies $(25,26)$. Zahraee et al. compared symmetry of load-bearing between women with and without LBP (25), and Sadeghisani et al. investigated the loading pattern in men with chronic LBP in comparison to men who were healthy (26). However, no study has yet investigated symmetrical load applied on the legs during gait in LBP athletes who practice martial arts.

In the present study, no significant difference was observed between the two groups in walking speed, cadence, and stride time when they walked with the dominant limbs. This reveals that the patients walked nearly similar to healthy people with respect to spatiotemporal gait pattern. These findings are different from those reported by Khodadade et al., Keefer, and Hill, who observed that patients with LBP tend to walk slower than healthy people. But, in Zahraee et al. study, similar findings were achieved (25). They observed that women with LBP walk similar to healthy women with regard to spatiotemporal parameters. Our results are also consistent with those obtained in Zahraee et al. study; when ASI of loads applied on the legs were compared between the groups, no significant difference was observed.

Childs et al. demonstrated that people with LBP stood with more asymmetry of weightbearing in bilateral lower limbs compared with healthy individuals (22), while Burnett et al. demonstrated that healthy individuals walk with symmetrical load bearing pattern (21). So, we hypothesized that asymmetrical loading on the legs could be demonstrated in people with LBP, especially athletes who practice martial arts. But, the results of our study did not prove the hypothesis, because no significant difference was observed in the amount of ASI of the loads applied on the legs between the groups. Although, the exact reasons for obtaining these results are not obvious, some arguments can be considered.

Zahraee et al. argued that, based on avoidanceendurance model, patients with LBP can ignore their pain and participate in physical performance without any limitation (25). This could have been a reason to observe no difference between the groups. In addition, our patients' pain intensity and level of disability were low, which could have affected the results; it is not clear whether similar results could be achieved with patients with higher pain intensity and disability. Furthermore, participants' fatigue might have affected the pattern of loading on the legs. All in all, no significant difference was observed in the pattern of forces applied on the legs between the two groups. Based on the results obtained in the present study, we also need to examine other mechanical risk factors which may be related to LBP in patients who practice martial arts sports activities.

The present study suffers from some limitations, which should be considered prior to generalizing the findings. First, we evaluated pattern of loading only during gait. Future studies are recommended to investigate pattern of loading during other activities, too. Secondly, only men were included in our study and no study, to the best of our knowledge, has yet examined the differences in symmetrical loadbearing between men and women. Thirdly, the current study failed to consider the healthy participants' sport activities. Specific activities are effective on mechanical variables (10), which may be task specific. These limitations should be addressed in future researches.

\section{CONCLUSION}

Based on the obtained results, it can be concluded that martial arts athletes with LBP apply symmetrical loads on the lower extremities during gait similar to healthy people.

\section{APPLICABLE REMARKS}

- Asymmetrical loading on the limbs applied by athletes who practice martial arts may be unrelated to their LBP symptoms.

- Other biomechanical risk factors which may be related to the problem of martial arts athletes must be examined carefully. 


\section{REFERENCES}

1. Dunn KM, Croft PR. Epidemiology and natural history of low back pain. Europa medicophysica. 2004;40(1):9-13.

2. Hoy D, Brooks P, Blyth F, Buchbinder R. The Epidemiology of low back pain. Best practice \& research Clinical rheumatology. 2010;24(6):769-81.

3. Andersson GB. Epidemiological features of chronic low-back pain. Lancet. 1999;354(9178):581-5.

4. Harvey J, Tanner S. Low back pain in young athletes. Sports Medicine. 1991;12(6):394-406.

5. Mousavi SJ, Akbari ME, Mehdian H, Mobini B, Montazeri A, Akbarnia B, et al. Low back pain in Iran: a growing need to adapt and implement evidence-based practice in developing countries. Spine (Phila Pa 1976). 2011;36(10):E638-46.

6. Meissam Sadeghisani MJS, Ahmad Reza Rafiei,, Reza Salehi. Pain, Disability, Fear-avoidance and Habitual Physical Activity in Subjects with Low Back Pain with and without Trunk and Hips Rotational Demand Sport Activities. Journal of Research in Rehabilitation Sciences. 2014:1213-21.

7. Diamond S, Borenstein D. Chronic low back pain in a working-age adult. Best practice \& research Clinical rheumatology. 2006;20(4):707-20.

8. Burnett AF, Cornelius MW, Dankaerts W, O'Sullivan P B. Spinal kinematics and trunk muscle activity in cyclists: a comparison between healthy controls and non-specific chronic low back pain subjects-a pilot investigation. Man Ther. 2004;9(4):211-9.

9. Luomajoki H, Kool J, de Bruin ED, Airaksinen O. Movement control tests of the low back; evaluation of the difference between patients with low back pain and healthy controls. BMC Musculoskelet Disord. 2008;9:170.

10. Sadeghisani M, Namnik N, Karimi MT, Rafiei AR, Manshadi FD, Eivazi M, et al. Evaluation of differences between two groups of low back pain patients with and without rotational demand activities based on hip and lumbopelvic movement patterns. Ortop Traumatol Rehabil. 2015;17(1):51-7.

11. Scholtes SA, Gombatto SP, Van Dillen LR. Differences in lumbopelvic motion between people with and people without low back pain during two lower limb movement tests. Clin Biomech (Bristol, Avon). 2009;24(1):7-12.

12. Murray E BE, Twycross-Lewis R, Morrissey D. The relationship between hip rotation range of movement and low back pain prevalence in amateur golfers. Phys Ther Sport. 2009;10(4):131-5.

13. Vad VB, Bhat AL, Basrai D, Gebeh A, Aspergren DD, Andrews JR. Low back pain in professional golfers: the role of associated hip and low back range-of-motion deficits. Am J Sports Med. 2004;32(2):494-7.

14. Van Dillen LR, Bloom NJ, Gombatto SP, Susco TM. Hip rotation range of motion in people with and without low back pain who participate in rotation-related sports. Phys Ther Sport. 2008;9(2):72-81.

15. Sahrmann S. Diagnosis and Treatment on Movement Impairment Syndromes. St Louis: MO:Mosby; 2002.

16. Rezaee M, Ghasemi M. Prevalence of low back pain among nurses: predisposing factors and role of work place violence. Trauma monthly. 2014;19(4):e17926.

17. Mohseni Bandpei MA, Ehsani F, Behtash H, Ghanipour M. Occupational low back pain in primary and high school teachers: prevalence and associated factors. Journal of manipulative and physiological therapeutics. 2014;37(9):702-8.

18. Biglarian A, Seifi B, Bakhshi E, Mohammad K, Rahgozar M, Karimlou M, et al. Low back pain prevalence and associated factors in Iranian population: findings from the national health survey. Pain research and treatment. 2012;2012:653060.

19. Mohseni-Bandpei MA, Ahmad-Shirvani M, Golbabaei N, Behtash H, Shahinfar Z, Fernandez-de-las-Penas C. Prevalence and risk factors associated with low back pain in Iranian surgeons. Journal of manipulative and physiological therapeutics. 2011;34(6):362-70.

20. Bono CM. Low-back pain in athletes. JBJS. 2004;86(2):382-96.

21. Burnett DR, Campbell-Kyureghyan NH, Cerrito PB, Quesada PM. Symmetry of ground reaction forces and muscle activity in asymptomatic subjects during walking, sit-to-stand, and stand-to-sit tasks. Journal of electromyography and kinesiology : official journal of the International Society of Electrophysiological Kinesiology. 2011;21(4):610-5.

22. Childs JD, Piva SR, Erhard RE, Hicks G. Side-to-side weight-bearing asymmetry in subjects with low back pain. Man Ther. 2003;8(3):166-9.

23. Duffell LD, Gulati V, Southgate DF, McGregor AH. Measuring body weight distribution during sit-to-stand in patients with early knee osteoarthritis. Gait Posture. 2013;38(4):745-50.

24. SHAFIZADEGAN Z, KARIMI MT, SHAFIZADEGAN F, REZAEIAN ZS. EVALUATION OF GROUND REACTION FORCES IN PATIENTS WITH VARIOUS SEVERITIES OF KNEE OSTEOARTHRITIS. Journal of Mechanics in Medicine and Biology. 2015:1650003.

25. Zahraee MH, Karimi MT, Mostamand J, Fatoye F. Analysis of asymmetry of the forces applied on the lower limb in subjects with nonspecific chronic low back pain. Biomed Res Int. 2014;2014:289491. 
26. Sadeghisani M, Sobhani V, Kabir MM, Asnaashari A, Rahmani P, Azimi H. Investigation of Weight-Bearing Symmetry in a Group of Athletes with Low Back Pain and Healthy People During Gait. Journal of Mechanics in Medicine and Biology. 2017;17(05):1750086.

27. Pieter W. Martial arts injuries. Epidemiology of pediatric sports injuries. 48: Karger Publishers; 2005. p. 59-73.

28. Sheikhhoseini R, O'Sullivan K, Alizadeh MH, Sadeghisani M. Altered Motor Control in Athletes with Low Back Pain: a Literature Review. Annals of Applied Sport Science. 2016;4(4):43-50.

29. Krismer M, Van Tulder M. Low back pain (non-specific). Best Practice \& Research Clinical Rheumatology. 2007;21(1):77-91.

30. Magee DJ. Orthopedic Physical Assessment-E-Book: Elsevier Health Sciences; 2014.

31. Herzog W, Nigg BM, Read LJ, Olsson E. Asymmetries in ground reaction force patterns in normal human gait. Med Sci Sports Exerc. 1989;21(1):110-4.

32. Adams MB, N.; Burton, K.; Dolan, P. The Biomechanics of Back Pain. Edinburgh,England: Churchill Livingstone; 2002.

33. McGill SM. The biomechanics of low back injury: implications on current practice in industry and the clinic. $\mathrm{J}$ Biomech. 1997;30(5):465-75.

34. Gombatto SP, Norton BJ, Scholtes SA, Van Dillen LR. Differences in symmetry of lumbar region passive tissue characteristics between people with and people without low back pain. Clin Biomech (Bristol, Avon). 2008;23(8):986-95.

35. Lund T, Nydegger T, Schlenzka D, Oxland TR. Three-dimensional motion patterns during active bending in patients with chronic low back pain. Spine (Phila Pa 1976). 2002;27(17):1865-74.

36. Gomez TT. Symmetry of lumbar rotation and lateral flexion range of motion and isometric strength in subjects with and without low back pain. J Orthop Sports Phys Ther. 1994;19(1):42-8. 